\section{Repurposing CAR T cells to treat SLE}

An improved understanding of pathogenesis in systemic lupus erythematosus (SLE) has not always translated to advances in patient treatment. Now, Marko Radic and colleagues report that CD19-specific chimeric antigen receptor (CAR) T cells, usually a therapy for patients with leukaemia, can be successfully used to treat SLE in mouse models.

"We used a CAR T cell approach to deplete B cells in mice to test how crucial B cells are in the pathogenesis of lupus," explains Radic. "This approach was attractive because, if successful, it might offer a viable new treatment option for lupus." Using retroviral transfection, the researchers inserted a CD19-targeted CAR into purified $\mathrm{CD}^{+} \mathrm{T}$ cells. This CAR included the variable fragment (Fv) of the CD19-specific 1D3 antibody, as a single-chain heterodimer of the light and heavy chains (scFv), the CD28 transmembrane and cytoplasmic signalling domains, and a $\mathrm{CD} 3 \zeta \mathrm{C}$ terminus engineered to prevent $\mathrm{T}$ cell exhaustion.

In two mouse models of lupus (NZB/W and MRL-lpr strains), CAR T cell transfer induced efficient and stable B cell depletion. This effect was accompanied by marked improvements in several measures of disease severity, including the elimination of circulating anti-DNA antibodies, as well as a significant increase in lifespan.

An analysis of immunoglobulin light chain transcript levels indicated that, following CAR T cell transfer, some $B$ cells persisted in the spleen and bone marrow but not in the kidney. Moreover, the treatment prevented the development of glomerulonephritis, as indicated by a reduction in proteinuria and, in the glomerulus, by a decrease in both cellular infiltration and IgG deposition.

" $\mathrm{B}$ cell depletion with rituximab was not effective in patients with SLE but CD19-targeted CAR T cells might prove to be more successful as they induce permanent and sustained B cell depletion," remarks Radic. "We are confident that our research paves the way for the use of CAR T cells to treat patients with severe lupus."

Monica Wang

ORIGINAL ARTICLE Kansal, R. et al. Sustained B cell depletion by CD19-targeted CAR T cells is a highly effective treatment for murine lupus. Sci. Transl Med. 11, eaav1648 (2019)

\title{
ORGANOIDS
}

\section{Tubuloids for modelling kidney disease}

Kidney organoids that aggregate in response to developmental cues have been generated from embryonic and induced pluripotent stem cells (PSCs). Using a modified protocol originally developed for the expansion of adult intestinal stem cells, researchers now show that kidney tubular epithelial organoids can be established from kidney tissue, as well as from urine. These so-called tubuloids can be used to model infectious, malignant and genetic kidney diseases. "The ability to culture tubular epithelium in this way is in line with the fact that adult stem cell-derived organoids recapitulate organ regeneration rather than development: tubule epithelium readily regenerates in vivo, whereas glomeruli do not regenerate easily," explains Frans Schutgens. "This system complements PSC-derived organoids, which model development and therefore include glomeruli."

To establish tubuloids the researchers used digested tubular fragments from human and mouse kidney. The tubuloids could be maintained in culture for $>6$ months and demonstrated chromosomal stability. Gene expression analysis showed that the tubuloids predominantly expressed markers of proximal tubule cells, but cells representative of collecting duct and loop of Henle cells were also present.

The researchers then used tubuloids to model three kidney diseases. Infection of the tubuloids with $B K$ virus induced enlargement of tubuloid nuclei, typical of BK virus nephropathy. Tubuloid infection was also induced by administration of infected urine. Tumour tubuloids were also established from two patients with Wilms tumour, and finally, tubuloids were established from the urine of a patient with cystic fibrosis, negating the need for nephrectomy or biopsy. Schutgens says that urine-derived tubuloids could represent an accessible approach to establish cultures from patients with tubular kidney diseases, while the ability to generate tubuloids from tumour and matching normal tissue will enhance understanding of different types of kidney cancer.

Susan J. Allison

ORIGINAL ARTICLE Schutgens, F. et al. Tubuloids derived from human adult kidney and urine for personalized disease modeling. Nat. Biotechnol. 37, 303-313 (2019)
ORGANOIDS

\section{A materials approach for kidney organoids}

Several protocols have been developed for generating kidney organoids from pluripotent stem cells (PSCs). Now, Nuria Montserrat and colleagues describe a protocol for the production of kidney organoids that transcriptomically resemble second trimester human fetal kidneys. They also show that use of a soft hydrogel promotes the differentiation of kidney organoid compartments. "One of our main goals has been to gain fundamental knowledge about the generation of renal-derived cells from human PSCs," says Montserrat. "In order to better mimic the environment of differentiating nephron progenitor cells in vivo, we lengthened the time human PSCs were exposed to a 3D microenvironment, thereby forcing cell-to-cell and cell-to-extracellular matrix contact."

This approach, combined with the administration of inductive cues, led to the derivation of kidney organoids with multiple nephron-like structures. A comparison of the transcriptional profile of day 16 organoids indicated they matched that of human fetal kidneys at 22 weeks gestation.

To improve the vascularization of the kidney organoids, the researchers implanted organoids into chick chorioallantoic membranes (CAMs). "Previous studies have reported vascularization of kidney organoids following transplantation under the kidney capsule of immunodeficient mice," says Montserrat. "CAMs are a highly vascularized tissue that represents a naturally inmmunodeficient environment. After transplantation, the induced glomerular-like structures became vascularized and acquired morphological features, such as slit diaphragmlike structures and secondary foot processes, indicating further differentiation." Montserrat and colleagues then fabricated polyacrylamide hydrogels that mimicked the biophysical properties of the CAM. "Using soft hydrogels during the first steps of PSC differentiation generated a higher number of nephron progenitors and nephrons than conventional approaches," she explains. "These hydrogels represent an affordable approach to further advance the field of organoid engineering."

\section{Susan J. Allison}

ORIGINAL ARTICLE Garreta, E. et al. Fine tuning the extracellular environment accelerates the derivation of kidney organoids from human pluripotent stem cells. Nat. Mater. https://doi.org/10.1038/s41563-019-0287-6 (2019) 